\title{
Depression and Insulin Resistance in Non-Diabetic Subjects: An Intervention Study with Insulin Clamp Technique
}

\author{
Shaheen Asghar ${ }^{1}$, A. Magnusson ${ }^{2}$, Akhtar Hussain $^{1 *}$, Lien M. Diep ${ }^{1,3}$, Bishwajit Bhowmik $^{1}$, \\ Per M. Thorsby ${ }^{3}$ \\ ${ }^{1}$ Institute of Health and Society, Section for International Health, University of Oslo, Oslo, Norway; ${ }^{2}$ National University Hospital \\ (Psychiatry), Reykjavík, Iceland; ${ }^{3}$ Hormone Laboratory, Department of Endocrinology, Oslo University Hospital, Oslo, Norway. \\ Email: " akhtar.hussain@medisin.uio.no
}

Received September $10^{\text {th }}, 2012$; revised October $24^{\text {th }}, 2012$; accepted November $24^{\text {th }}, 2012$

\begin{abstract}
Aims: To clarify the relationship between depression and glucose metabolism using sensitive measures of insulin resistance, and to assess if remission of depression results in enhanced insulin sensitivity. Methods: An intervention study to quantify changes in insulin sensitivity before and after treatment of depression was carried out. Twenty six Pakistani women with newly diagnosed depression underwent euglycemic insulin clamp to measure insulin sensitivity at inclusion and again after treatment of depression $6-8$ weeks later. Twenty-three individuals completed both tests. Results: Significant improvement of insulin sensitivity was observed following the treatment of depression. The improved insulin sensitivity remained statistically significant after controlling for confounding factors. Conclusions: This study establishes a relationship between depression and insulin resistance. It demonstrated that insulin sensitivity can be improved by treating depression.
\end{abstract}

Keywords: Clamp Technique; Depression; Insulin Resistance

\section{Introduction}

Depression, one of the leading causes of disease today [1], is associated with poor glycemic control [2], diabetes-related complications [3], poor quality-of-life [4] and increased mortality in diabetic patients $[5,6]$.

Diabetes and depression are both commonly occurring conditions that are associated with the modern lifestyles in today's society. The total number of patients with diabetes is projected to rise from 171 million in 2000 to 366 million in 2030 [7]. Adults with depression have a $37 \%$ increased risk of developing type 2 diabetes [8] and evidence from cross-sectional and prospective studies suggests that depressive symptoms negatively influence glucose metabolism $[9,10]$. Studies have shown that diabetes is twice as likely to develop in depressed individuals compared with non-depressed individuals $[11,12]$. Depression may contribute to metabolic abnormalities preceding the development of diabetes [13], although the evidence is still conflicting.

One study demonstrated that depressed patients with normal glucose tolerance had a higher degree of insulin resistance relative to non-depressed control subjects [14]

"Corresponding author. while another study found that the prevalence of depression was lowest at the highest levels of insulin resistance among non-diabetic women [15]. Thus, it is important to clarify the relationship between depression and insulin resistance. More specifically whether depression may cause insulin resistance. Most studies rely on relatively insensitive methods or approximations for assessing insulin resistance. Furthermore, the evidence comes primarily from association studies. There have been to intervention studies to date that have assessed the relationship between depression and insulin sensitivity. In this report we employ an intervention study design to quantify changes in insulin sensitivity before and after treatment of depression with a sensitive method known as hyperinsulinemic clamp [16]. We hypothesized that a reduction in depressive symptoms would lead to a better insulin sensitivity, and possibly changes in other parameters of the metabolic syndrome.

\section{Method}

\subsection{Study Population}

Thirty women newly diagnosed with major depression of Pakistani orgin were invited into the study. Participants 
were recruited from a private clinic for depression (Depresjonsklinikken, Eckersbergsgate 29, 0266 Oslo) in collaboration with, Aker University Hospital. Patients were recruited from newly diagnosed depressed women originating from Pakistan, but living in Oslo, Norway. The rational for choosing this inclusion criteria is to avoid influence of ethnicity and gender in relation to insulin sensitivity and the risk of diabetes. Owing to the laborious technique required to apply clamp technique to assess insulin sensitivity or insulin resistance, it would not be possible to employ this procedure on a larger sample, which may have allowed us to do stratified analysis.

Inclusion criteria were as follow:

A clinical diagnosis of major depression by a psychiatrist specializing in mood disorders ( $1^{\text {st }}$ author):

- Age 20 to 70 years;

- Women of Pakistani Ethnic origin;

- A depressive symptom score $>20$ on the MADRS [17];

- The patient understood the protocol and was able to give an informed consent;

- The patient's condition allowed him to carry out the glucose clamp experiment;

- The depression was not judged to be primarily triggered by an adverse life event.

Exclusion criteria:

- Diabetes or history of gestation diabetes;

- Pregnancy;

- A serious psychotic depression;

- Schizophrenia or an obvious personality disorder (but other psychiatric co-morbid diagnoses were allowed).

The participants were oriented of the research objects and procedures prior to inclusion and they signed an informed consent. Twenty six of 30 patients agreed to participate $(87 \%)$ and 23 of those completed the study.

\subsection{Assessment of Depressive Symptoms}

All the patients received a clinical diagnosis of depression, based on ICD 10 , by an experienced psychiatrist $\left(1^{\text {st }}\right.$ author). In addition, the Montogomery-Aasberg Depression Rating Scale (MADRS) was employed to assess depressive symptoms [17] and was administered to all individuals by the same psychiatrist. The MADRS has 10 items; the response to each item ranges from 0 to 6 , the sum score can thus range from 0 to 60 . The cut-off score for a likely depression is considered $\geq 20$.

\subsection{Intervention}

The depressed patients received standard clinical management by a psychiatrist ( $1^{\text {st }}$ author). All patients were started on ecitalopram $10 \mathrm{mg}$ with dose increments until clinical response was achieved. One patient who started on ecitalopram was switched to fluoxetin $(20 \mathrm{mg})$ due to the clinical situation. The average daily dose was $20 \mathrm{mg}$ ecitalopram. The patients were treated for 6 to 8 weeks.

Insulin resistance was measured at two time points; first at the time of diagnosis of depression, after verifying a MADRS score $>20$, and again after remission of depression to at least MADRS $\leq 10$ ). For each subject the measurements were made at the same time of the day, with the same study nurses, and at the same Hormone Laboratory, Aker University Hospital.

\subsubsection{The Hyperinsulinemic Euglycemic Clamp Technique for Quantifying Insulin Resistance}

With the hyperinsulinemic euglycemic clamp technique [16], the plasma insulin concentration is acutely raised by giving a standard continuous infusion of insulin according to weight $(0.3 \mathrm{mU} / \mathrm{kg})$ and maintained during the entire investigation. The plasma glucose concentration is held constant at basal levels by a variable glucose infusion using the negative feedback principle. Insulin is infused via the antecubital catheter along with the glucose infuscate. The computation for the periodic adjustments in the glucose infusion is made every $5 \mathrm{~min}$ : if the actual glucose concentration is higher than the goal, the infusion is decreased and vice versa.

Under these steady-state conditions of hyperinsulinemia and euglycemia, the pancreatic insulin production is suppressed and glucose infusion rate equals glucose uptake in the body and is therefore a measure of tissue sensitivity to exogenous insulin. The measurement of the glucose uptake is done in the last 30 minutes of the clamp and calculated as $\mu \mathrm{g} / \mathrm{kg} / \mathrm{min} / \mathrm{ins}$. This measure is called: "The Glucose Disposal Rate per inulin (GDRI)".

\subsubsection{Blood Tests}

After an overnight fast of eight hours, fasting $10 \mathrm{ml}$. Venous blood was collected from each participant at base line for fasting blood glucose, insulin and cortisol. An oral glucose tolerance test was performed on each individual with $75 \mathrm{~g}$ of glucose and a new blood was drawn after two hours to determine the plasma glucose values (OGTT).

Fasting blood glucose (FBG) and oral glucose tolerance test (OGTT) were analyzed by glucose oxidase method (Randox, UK) for the diagnosis of diabetes mellitus. Diabetes was diagnosed if FPG value was $\geq 7.0$ $\mathrm{mmol} / \mathrm{l}$ and/or 2-h post glucose was $\geq 11.1 \mathrm{mmol} / \mathrm{l}$.

Blood samples were drawn and analysed for plasma glucose during clamp with the glucose oxidase method using a Glucose Analyser II (Beckman Instruments, Fullerton, CA, USA). The serum levels of insulin were measured by radioimmunoassay (RIA) (Linco Researc, Inc, St. 
Charles, MO, USA) with intra-assay coefficient of variation $<5 \%$. Cortisol in serum were measured with competitive luminoimmunoassay (DPC, Los Angeles, CA, USA) with intra-assay coefficient of variation $<7 \%$.

\subsection{Power Analysis}

A difference of insulin sensitivity of $15 \%$ before and after intervention, would require 30 subjects to have an $80 \%$ power $(\beta-20)$ to identify a difference at 0.05 level of statistical significance in parallel groups.

\subsection{Statistical Analysis}

Comparisons of group differences were analysed in pair with student's $t$ test. Mixed model multivariate linear regression was applied to adjust for potential confounding factors of insulin resistance. All statistical analyses were done with SPSS 16.0 (SPSS Inc., Chicago, IL, USA). All p-values presented are two tailed and confidence intervals are at $95 \%$.

\section{Results}

Table 1 shows that after remission of depression, statistically significant differences were observed for the mean insulin sensitivity (GDRI) $\mathrm{p}=0.02,95 \% \mathrm{CI}(-0.28-3.35)$, fasting cortisol, $\mathrm{p}=0.01,95 \% \mathrm{CI}(-44.6--162.8)$ and fasting insulin, $\mathrm{p}<0.01,95 \% \mathrm{CI}(-49.1-3.15)$.

Cortisol, waist circumference and BMI may all effect insulin sensitivity [18], but owing to limited sample size and high interaction between waist and BMI we have entered cortisol and BMI together with the MADRS score into a multivariate linear regression on the GDRI to examine if depression did affect insulin sensitivity (GDRI) independent of these factors (Table 2). There was a statistically significant improvement of insulin resistance (GDR) with remission of depression independent of BMI and fasting cortisol levels ( $\mathrm{p}<0.09,95 \% \mathrm{CI}$ : $-2.18-0.15$, Table 2). On average the GDR was reduced by 0.06 units for a reduction of one unit of depression (p $=0.049$ ). For the reduction of each unit of central obesity (waist, cm), fasting cortisol (pmol/l) and BMI $\left(\mathrm{kg} / \mathrm{m}^{2}\right)$, GDR was reduced by $0.02,0.007$ and $0.03 \mu \mathrm{g} / \mathrm{kg} / \mathrm{min} / \mathrm{ins}$ respectively.

A multivariate linear regression of changes in insulin resistance from pre and post-treatment of depression (Figure 1) reveals an improvement in insulin sensitivity (GDR). Figure 2 demonstrates the association between insulin sensitivity and depressive symptoms. Insulin sensitivity appears to be closely related to depressive symptoms and a decline with older individuals.

\section{Discussion}

These data showed that insulin sensitivity was related to the severity of depression, and that treatment of depression resulted in improved insulin sensitivity. This effect of depression on insulin resistance persisted even after adjusting for various possible confounding factors. To our knowledge, this is one of the first intervention studies to examine the association between depression and insulin resistance in depressed and remitted state.

We have previously shown an association between diabetes and depression in newly diagnosed patients with diabetes in Bangladesh [19] and Pakistan [20,21]. There is a growing body of evidence that depression is associated

Table 1. Significant changes in mean insulin sensitivity (GDRI), fasting cortisol and fasting insulin after remission of depression.

\begin{tabular}{|c|c|c|c|c|c|c|}
\hline & \multirow{2}{*}{ Mean, depressed } & \multirow{2}{*}{ Mean, remitted } & \multirow{2}{*}{$\begin{array}{l}\text { Difference } \\
\text { remitted-depressed }\end{array}$} & \multicolumn{2}{|c|}{$95 \%$ CI of mean diff. } & \multirow{2}{*}{$\begin{array}{l}\text { p-value for the } \\
\text { difference }\end{array}$} \\
\hline & & & & lower & upper & \\
\hline BMI $\left(\mathrm{kg} / \mathrm{m}^{2}\right)$ & 27.39 & 27.45 & 0.06 & -0.20 & 0.31 & 0.64 \\
\hline Waist (cm) & 87.17 & 86.44 & -0.72 & -1.61 & 0.16 & 0.10 \\
\hline $\begin{array}{l}\text { Insulin sensitivity (GDR) } \\
\qquad(\mu \mathrm{g} / \mathrm{kg} / \mathrm{min} / \text { ins })\end{array}$ & 4.81 & 6.96 & 2.15 & 1.49 & 2.81 & 0.02 \\
\hline Depression score MADRS & 30.50 & 6.94 & -23.55 & -25.62 & -21.49 & 0.00 \\
\hline Fasting insulin (pmol/1) & 65.72 & 47.22 & -18.50 & -35.97 & -1.03 & 0.04 \\
\hline Fasting cortisol (pmol/1) & 327.50 & 217.67 & -109.83 & -167.16 & -52.51 & 0.01 \\
\hline Fasting plasma glucose $(\mathrm{mM} / \mathrm{L})$ & 5.61 & 5.37 & -0.24 & -0.44 & -0.03 & 0.03 \\
\hline OGTT & 7.12 & 7.00 & -0.12 & -0.20 & -0.04 & 0.07 \\
\hline DBP (mmhg) & 69.39 & 67.44 & -1.94 & -5.64 & 1.75 & 0.28 \\
\hline SBP (mmhg) & 116.39 & 113.89 & -2.50 & -7.91 & 2.91 & 0.34 \\
\hline
\end{tabular}


Table 2. Multivariate linear regressions of changes in insulin resistance from its initial value at inclusion (while depressed) to $2^{\text {nd }}$ investigation i.e. after recovery from depressive symptoms in a mixed model with depression, cortisol and BMI.

\begin{tabular}{|c|c|c|c|c|c|}
\hline \multirow{2}{*}{ Change of parameters } & \multirow{2}{*}{ Estimates } & \multirow{2}{*}{$\mathrm{t}$} & \multirow{2}{*}{ sig } & \multicolumn{2}{|c|}{$95 \%$ Confidence Interval } \\
\hline & & & & Lowe Bound & Upper Bound \\
\hline Depression (MADRS) & -0.06 & -1.94 & 0.05 & -0.12 & 0.007 \\
\hline Cortisol & -0.006 & -1.86 & 0.055 & -0.01 & 0.002 \\
\hline BMI & -0.24 & -2.85 & 0.005 & -0.42 & -0.10 \\
\hline
\end{tabular}

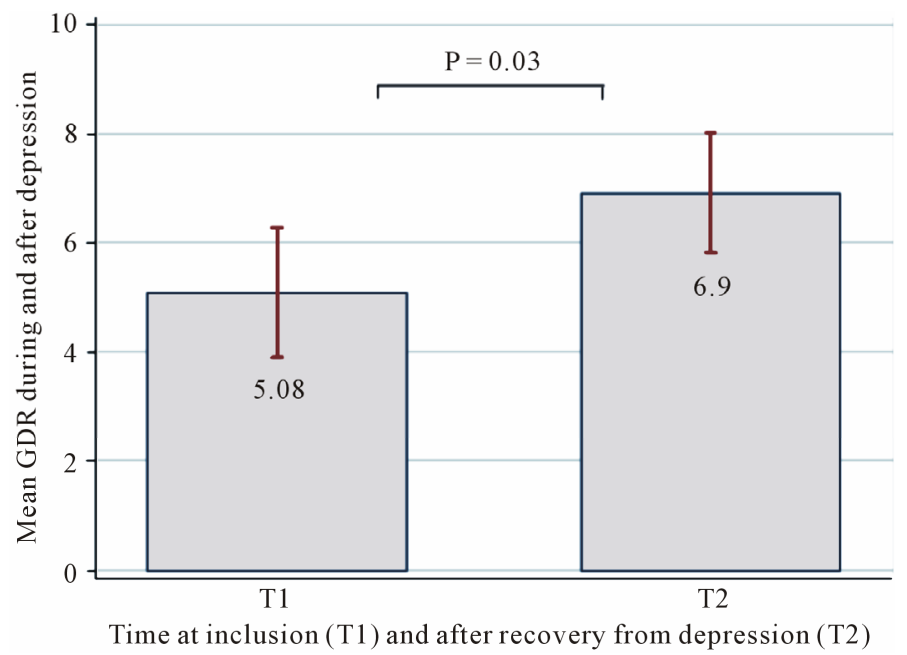

Figure 1. Shows the improvement in insulin sensitivity (GDR) from depressed state to remission of depression.

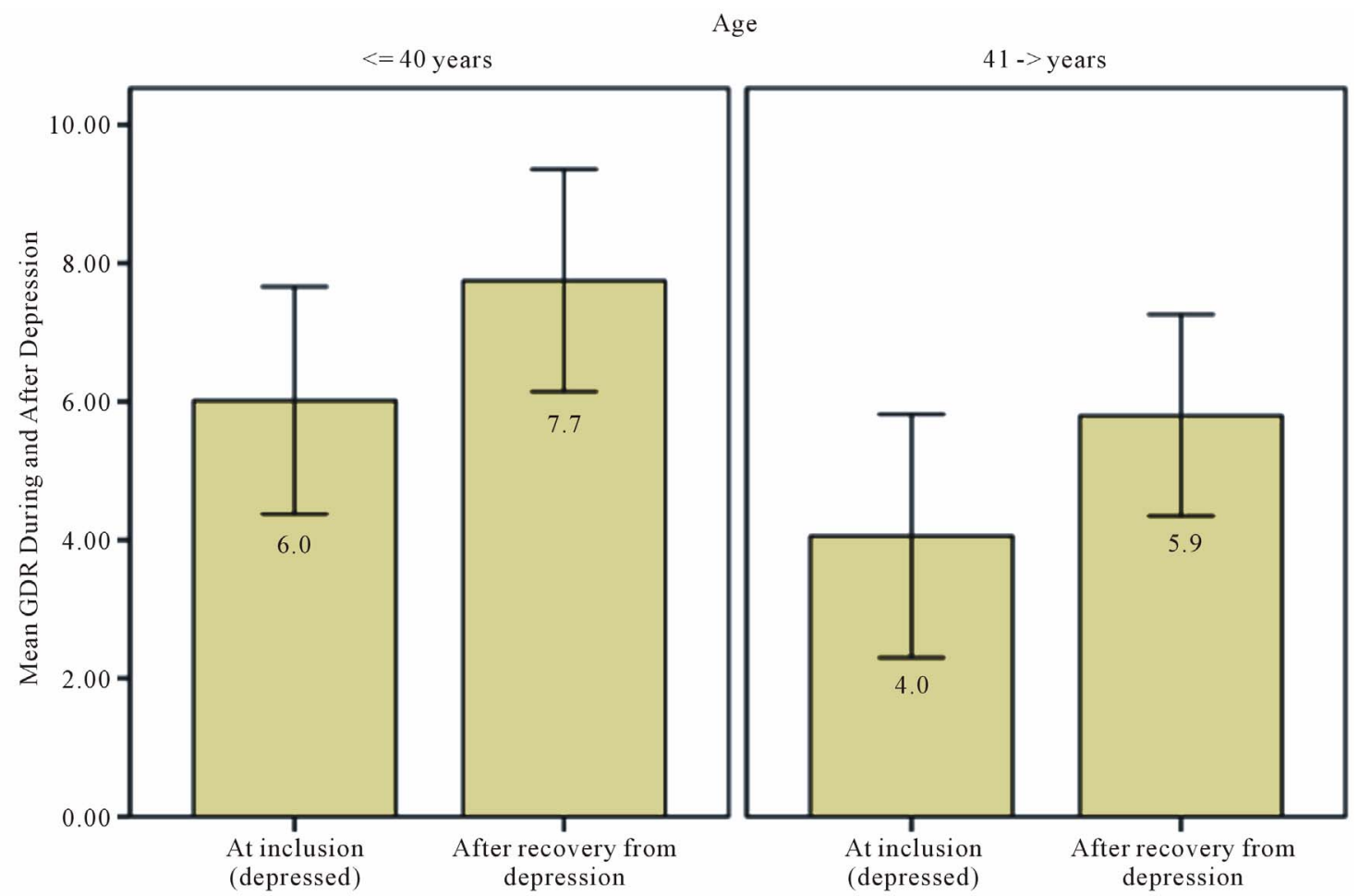

Figure 2. Shows the association between insulin sensitivity and depressive symptoms, both baseline and remission values are included. 
with increased risk of diabetes mellitus [22,23]. The underlying mechanisms, however, are still poorly understood. Insulin resistance, which plays a pivotal role in the pathogenesis of type 2 diabetes [24], has been suggested to be the underlying tie between depression and diabetes [25].

One of the suggested links between depression and diabetes is cortisol. Cortisol plays a major role in regulating many of the body's homeostatic functions, including blood pressure, blood-lipid profile, immune- and inflammatory responses, fat distribution and importantly, glucose metabolism and insulin resistance [26]. Cortisol has been shown to be responsive to emotional stress [27], and higher levels have been observed in depressed individuals [28]. Furthermore, circadian control of cortisol secretion is perturbed in depression [29].

In this study we found that fasting cortisol levels were significantly lower after remission of depressive symptoms. This is in accordance with several other studies [30]. Changes in blood cortisol concentrations with levels of depression might have explained some of the effect of depressive symptoms on insulin sensitivity in our study. However, the observed association between remission of depressive symptoms and improved insulin sensitivity remained statistically significant even after cortisol levels were adjusted in the regression model. Thus the effect of depressive symptoms on insulin resistance may either have been mediated by factors known to effect insulin sensitivity for which we did not control, such as pro-inflammatory cytokines $[31,32]$ and essential fatty acids [33]; or to hitherto unknown novel mechanisms.

It is less likely that the SSRIs used in this study had in themselves an enhancing effect on insulin sensitivity. On the contrary, many antidepressives have weight gain as a side effect which would be expected to reduce insulin sensitivity [34]. We have not been able to identify studies that showed an improved insulin sensitivity by taking SSRIs. On the contrary (conversely), there are some studies linking SSRIs to risk factors for diabetes $[35,36]$. Of interest, a large epidemiological study showed that most individuals taking various SSRIs had elevated indices of metabolic syndrome, except for individuals on citalopram, which is the racemixture of ecitalopram used in the present study.

This is one of few intervention studies in the field. It utilized the hyperinsulinemic euglycemic clamp technique, the gold standard for measuring insulin sensitivity. To reduce variability, insulin sensitivity was measured in the same individual before and after remission of depressive symptoms, at the same time of the day, by the same study-nurse and all the participants were of the same gender and ethnicity. The euglycemic insulin clamp tech- nique is expensive, laborious, time consuming and taxing on the participants; hence the sample size was relatively modest in the study. However, the technique is very sensitive diminishing the need for a large sample.

There are a number of limitations: 1) The external validity of the results may be restricted owing to a small sample size which is less likely to secure the representativeness. However, due to the nature of human physiology, the findings may still be considered an important contribution; 2) The confounding effect cannot be completely overruled as we could not include all potential confounding factors in the multivariate model, due to the limited number of subjects who completed pre and post study.

\section{Conclusion}

Our data support the hypothesis that depression is associated with insulin resistance. Furthermore, insulin sensitivity was significantly improved by treating depressive symptoms. This effect of depressive symptoms on insulin sensitivity remained statistically significant even after several potential confounding factors had been controlled for. These results should be further tested on a larger sample to confirm our findings.

\section{Funding}

This study was fully funded by the Norwegian Eastern Health Region (Helse Øst) and the Norwegian Medical association. Dr. Asghar is a Research fellow at the faculty of Medicine, University of Oslo.

\section{Competing Interests}

All authors have completed the Unified Competing Interest form at www.icmje.org/coi_disclosure.pdf (available on request from the corresponding author) and declare: no support from any organization for the submitted work; no financial relationships with any organizations that might have an interest in the submitted work in the previous three years, no other relationships or activities that could appear to have influenced the submitted work.

\section{Acknowledgements}

We are particularly indebted to all the participants who endured pain to undergo euglycaemic clamp twice and sustained cooperation during the study. The two nurses (A. Halsne and G. Vinje) and the chief consultant Prof. Kaare Birkeland made an indispensable contribution in order to execute euglycaemic clamp to assess insulin resistance. The Faculty of Medicine, University of Oslo awarded the Research Fellowship. Health region (South East) of Norway and the Norwegian Medical Association 
funded the investigations.

\section{REFERENCES}

[1] T. B. Ustun, J. L. Ayuso-Mateos, S. Chatterji, et al., "Global Burden of Depressive Disorders in the Year 2000," The British Journal of Psychiatry, Vol. 184, 2000, pp. 386-392. doi:10.1192/bjp.184.5.386

[2] P. J. Lustman, R. J. Anderson, K. E. Freedland, et al., "Depression and Poor Glycemiccontrol: A Meta-Analytic Review of the Literature," Diabetes Care, Vol. 23, No. 7, 2000, pp. 934-942. doi:10.2337/diacare.23.7.934

[3] M. De Groot, R. Anderson, K. E. Freedland, et al., "Association of Depression and Diabetes Complications: A Meta-Analysis," Psychosomatic Medicine, Vol. 63, No. 4, 2001, pp. 619-630.

[4] D. J. Wexler, R. W. Grant, E. Wittenberg, et al., "Correlates of Health Related Quality of Life in Type 2 Diabetes," Diabetologia, Vol. 49, No. 7, 2006, pp. 1489-1497. doi:10.1007/s00125-006-0249-9

[5] L. E. Egede, P. J. Nietert and D. Zheng, "Depression and All-Cause and Coronary Heart Disease Mortality among Adults with and without Diabetes," Diabetes Care, Vol. 28, No. 6, 2005, pp. 1339-1345. doi:10.2337/diacare.28.6.1339

[6] X. Zhang, S. L. Norris, E. W. Gregg, et al., "Depressive Symptoms and Mortality among Persons with and without Diabetes," American Journal of Epidemiology, Vol. 161, No. 7, 2005, pp. 652-660.

doi:10.1093/aje/kwi089

[7] W. Sarah, R. Gojka, A. Green, et al., "Global Prevalence of Diabetes," Diabetes Care, Vol. 27, No. 5, 2004, pp. 1047-1053.

[8] M. J. Knol, J. W. R. Twisk, A. T. F. Beekman, et al., "Depression as a Risk Factor for the Onset of Type 2 Diabetes Mellitus. A Meta-Analysis," Diabetologia, Vol. 49, No. 5, 2006, pp. 837-845. doi:10.1007/s00125-006-0159-x

[9] J. M. McCaffery, R. Niaura, J. F. Todaro, et al., "Depressive Symptoms and Metabolic Risk in Adult Male Twins Enrolled in the National Heart, Lung, and Blood Institute Twin Study," Psychosomatic Medicine, Vol. 65, No. 3, 2003, pp. 490-497. doi:10.1097/01.PSY.0000041545.52924.82

[10] E. C. Suarez, "Sex Differences in the Relation of Depressive Symptoms, Hostility, and Anger Expression Indices of Glucose Metabolism in Non Diabetics," Health Psychol, Vol. 25, No. 4, 2006, pp. 484-492. doi:10.1037/0278-6133.25.4.484

[11] W. W. Eaton, H. Armenian, J. Gallo, et al., "Depression and Risk for Onset of Type 2 Diabetes: A Prospective Population-Based Study," Diabetes Care, Vol. 19, No. 10, 1996, pp. 1097-1102. doi:10.2337/diacare.19.10.1097

[12] N. Kawakami, N. Takatsuka, H. Shimizu, et al., "Depressive Symptoms and Occurrence of Type 2 Diabetes among Japanese Men," Diabetes Care, Vol. 22, No. 7, 1999, pp. 1071-1076. doi:10.2337/diacare.22.7.1071
[13] F. Talbot and A. Nouwen, "A Review of the Relationship between Depression and Diabetes in Adults: Is There a Link?" Diabetes Care, Vol. 23, No. 10, 2000, pp. 15561562. doi:10.2337/diacare.23.10.1556

[14] M. Chiba, S. Suzuki, Y. Hinokio, et al., "Tyrosine Hydroxylase Gene Microsatellite Polymorphism Associated with Insulin Resistance in Depressive Disorder," Metabolism, Vol. 49, No. 9, 2000, pp. 1145-1149. doi:10.1053/meta.2000.8611

[15] D. A. Lawlor, G. Davey Smith and S. Ebrahim, "Association of Insulin Resistance with Depression: Cross Sectional Findings from the British Women's Heart and Health Study," British Medical Journal, Vol. 327, 2003, pp. 1383-1384. doi:10.1136/bmj.327.7428.1383

[16] R. A. DeFronzo, J. D. Tobin and R. Andres, "Glucose Clamp Technique: A Method for Quantifying Insulin Secretion and Resistance," American Journal of Physiology, Vol. 237, No. 3, 1979, pp. E214-E223.

[17] M. Zimmerman, M. A. Posternak and I. Chelminski, "Derivation of a Definition of Remission on the Montgomery-Asberg Depression Rating Scale Corresponding to the Definition of Remission on the Hamilton Rating Scale for Depression," Journal of Psychiatric Research, Vol. 38, No. 6, 2004, pp. 577-582. doi:10.1016/j.jpsychires.2004.03.007

[18] A. Holmang and P. Bjorntrop, "The Effects of Cortisol on Insulin Sensitivity in Muscle," Acta Physiologica Scandinavica, Vol. 144, No. 4, 1992, pp. 425-431. doi:10.1111/j.1748-1716.1992.tb09316.x

[19] S. Asghar, A. Hussain, A. K. A. Khan, et al., "Prevalence of Depression and Diabetes: A Populationbased Study from Rural Bangladesh," Diabetic Medicine, Vol. 24, No. 8, 2007, pp. 872-877. doi:10.1111/j.1464-5491.2007.02136.x

[20] F. Fareeha, S. Asghar, A. Hussain, et al., "Depression and Diabetes in High-Risk Urban Population of Pakistan," The Open Diabetes Journal, Vol. 3, 2010, pp. 1-5. doi:10.2174/1876524601003010001

[21] N. Zahid, S. Asghar, A. Hussain, et al., "Depression and Diabetes in a Rural Community in Pakistan," Diabetes Research and Clinical Practice, Vol. 79, No. 1, 2008, pp. 124-127. doi:10.1016/j.diabres.2007.07.006

[22] R. J. Anderson, K. E. Freedland, R. E. Clouse, et al., "The Prevalence of Comorbid Depression in Adults with Diabetes: A Meta-Analysis," Diabetes Care, Vol. 24, No. 6, 2001, pp. 1069-1078. doi:10.2337/diacare.24.6.1069

[23] S. H. Golden, J. E. Williams, D. E. Ford, et al., "Depressive Symptoms and the Risk of Type 2 Diabetes: The Atherosclerosis Risk in Communities Study," Diabetes Care, Vol. 27, No. 2, 2004, pp. 429-435.

[24] American Diabetes Association, "Diagnosis and Classification of Diabetes Mellitus," Diabetes Care, Vol. 30, No. S1, 2007, pp. S42-S47. doi:10.2337/dc07-S042

[25] D. L. Musselman, E. Betan, H. Larsen, et al., "Relationship of Depression to Diabetes Types 1 and 2: Epidemiology, Biology, and Treatment," Biological Psychiatry, Vol. 54, No. 3, 2003, pp. 317-329. 
doi:10.1016/S0006-3223(03)00569-9

[26] J. M. Olefsky and G. Kimmerling, "Effects of Glucocorticoids on Carbohydrate Metabolism," American Journal of the Medical Sciences, Vol. 271, No. 2, 1976, pp. 202210. doi:10.1097/00000441-197603000-00009

[27] P. Linkowski, J. Mendlewicz, R. Leclercq, et al., "The 24-Hour Profile of Adrenocorticotropin and Cortisol in Major Depressive Illness," The Journal of Clinical Endocrinology \& Metabolism, Vol. 61, No. 3, 1985, pp. 429438. doi:10.1210/jcem-61-3-429

[28] J. F. Mortola, J. H. Liu, J. C. Gillin, et al., "Pulsatile Rhythms of Adrenocorticotropin (ACTH) and Cortisol in Women with Endogenous Depression: Evidence of Increased ACTH Pulse Frequency," The Journal of Clinical Endocrinology \& Metabolism, Vol. 65, No. 5, 1987, pp. 962-968. doi:10.1210/jcem-65-5-962

[29] P. Boyce and E. Barriball, "Circadian Rhythms and Depression," Australian Family Physician, Vol. 39, No. 5, 2010, pp. 307-310.

[30] M. S. McKay and K. K. Zakzanis, "The Impact of Treatment on HPA Axis Acitivity on Unipolar Major Depression," Journal of Psychiatric Research, Vol. 44, No. 3, 2010, pp. 183-192. doi:10.1016/j.jpsychires.2009.07.012

[31] A. Appels, F. W. Bar, J. Bar, et al., "Inflammation, Depressive Symptomatology, and Coronary Artery Disease,"
Psychosomatic Medicine, Vol. 62, No. 5, 2000, pp. 601-605.

[32] B. W. Penninx, S. B. Kritchevsky, K. Yaffe, et al., "Inflammatory Markers and Depressed Mood in Older Persons: Results from the Health, Aging and Body Composition Study," Biological Psychiatry, Vol. 54, No. 5, 2003, pp. 566-572. doi:10.1016/S0006-3223(02)01811-5

[33] P. K. Ranjekar, A. Hinge, M. V. Hegde, et al., "Decreased Antioxidant Enzymes and Membrane Essential Polyunsaturated Fatty Acids in Schizophrenic and Bipolar Mood Disorder Patients," Psychiatry Research, Vol. 121, No. 2, 2003, pp. 109-122. doi:10.1016/S0165-1781(03)00220-8

[34] A. Serretti and L. Mandelli, "Antidepressants and Body Weight: A Comprehensive Review and Meta-Analysis," Journal of Clinical Psychiatry, Vol. 71, No. 10, 2010, pp. 1259-1272. doi:10.4088/JCP.09r05346blu

[35] M. B. Raeder, I. Bjelland, V. S. Emil, et al., "Obesity, Dyslipidemia, and Diabetes with Selective Serotonin Reuptake Inhibitors: The Hordaland Health Study," Journal of Clinical Psychiatry, Vol. 67, No. 12, 2006, pp. 1974 1982. doi:10.4088/JCP.v67n1219

[36] C. Lauren, Brown, R. M. Sumit, et al., "Type of Antidepressant Therapy and Risk of Type 2 Diabetes in People with Depression," Diabetes Research and Clinical Practice, Vol. 79, No. 1, 2008, pp. 61-67. 